\title{
Body Composition Analysis of 10 Years versus 5 Years of Adjuvant Endocrine Therapy in Patients with Nonmetastatic Breast Cancer
}

\author{
Ruyi Hu (D), Xinran Cheng $(\mathbb{D}$, Jun Liu $(\mathbb{D}$, Xu Lai $(\mathbb{D})$, Ruifeng Wang $(\mathbb{D}$, Dongchang Yu $(\mathbb{D}$, \\ Yanan Fan $(\mathbb{D}$, and Zhaoshi Yu (D)
}

Thyroid Breast Surgery Department, Hubei Ezhou Central Hospital, Ezhou, Hubei, China

Correspondence should be addressed to Zhaoshi Yu; zhaoshiyu31@gmail.com

Ruyi Hu and Xinran Cheng contributed equally to this work.

Received 3 November 2020; Revised 29 December 2020; Accepted 31 December 2020; Published 16 January 2021

Academic Editor: Dan Zhao

Copyright ( 2021 Ruyi Hu et al. This is an open access article distributed under the Creative Commons Attribution License, which permits unrestricted use, distribution, and reproduction in any medium, provided the original work is properly cited.

\begin{abstract}
Objective. Our study aims to investigate the association of extended adjuvant endocrine therapy with disease-free survival (DFS), muscle mass, muscle strength, and visceral adipose tissue in patients with nonmetastatic breast cancer and the effect of extended endocrine therapy on body composition. Patients and Methods. Patients $(N=90)$ with nonmetastatic breast cancer aged between 60 and 65 years old were prospectively recruited in this study, compromising a cohort of subjects rece iving 5 years or 10 years of adjuvant endocrine therapy. Patients' DFS was compared between these two groups. Patients' body composition including muscle and fat using CT scans, muscle strength, and gait speed was evaluated in these two groups. Results. Dietary behavior was recorded with the food frequency questionnaire (FFQ). Patients' age, body weight, and body mass index (BMI) did not differ between the two groups. An extended adjuvant endocrine therapy into 10 years could translate into DFS benefit (123.8 vs. 102.2 months, $P=0.038$ ). Patients receiving 10 years of adjuvant endocrine therapy had less skeletal muscle and more visceral fat compared with patients receiving 5 years of adjuvant endocrine therapy. The skeletal muscle index was $50.3 \pm 1.6 \mathrm{~cm}^{2} / \mathrm{m}^{2}$ versus $46.5 \pm 1.3 \mathrm{~cm}^{2} / \mathrm{m}^{2}$ in the 10 years or 5 years of adjuvant endocrine therapy group $(P=0.042)$. The visceral fat was $28.9 \pm 2.9 \mathrm{~cm}^{2} / \mathrm{m}^{2}$ versus $55.0 \pm 3.2 \mathrm{~cm}^{2} / \mathrm{m}^{2}$ in the 10 years or 5 years of adjuvant endocrine therapy group $(P=0.011)$. The muscle strength, gait speed, and FFQ results in the two groups not reaching statistical difference. Conclusion. In conclusion, breast cancer patients with 10 years of adjuvant endocrine therapy had DFS benefit, but with more muscle loss and adipose tissue deposits compared to patients receiving 5 years of adjuvant endocrine therapy.
\end{abstract}

\section{Introduction}

Breast cancer is the first most common malignancy in females worldwide [1]. The average 10-year survival rate for women with invasive breast cancer reaches 90\% [2]. Early breast cancer patients with the chance of surgery have a better survival rate. Adjuvant endocrine therapy undoubtedly extends the survival time [3-5]. Patients with early breast cancer are suggested to take at least 5 years of adjuvant endocrine therapy [5]. However, there is still inconsistency regarding the length of adjuvant endocrine therapy. Some oncologists recommend patients to extend endocrine therapy to 10 years to control the increased recurrence rate and mortality after patients have taken 5 years of adjuvant therapy. However, the study conclusions are controversial although several studies have published the survival data of comparison of early breast cancer patients receiving 5 years of adjuvant endocrine therapy in comparison with patients receiving 10 years of adjuvant endocrine therapy [6]. A previous clinical study of 30848 patients with breast cancer suggested that more than an extended 10year endocrine therapy yields a disease-free survival (DFS) benefit for patients with early-stage breast cancer. It is worth more clinical data about the survival benefits and adverse events before recommendations of extended adjuvant endocrine therapy were given.

The adverse events of endocrine therapy could be decreased muscle mass and increased fat tissue. Accelerated muscle loss, also called sarcopenia, has been proved to be correlated to the menopausal transition and thus linked to 
declining estrogen levels [7]. Estrogen may take part in muscle metabolism through estrogen receptors found on skeletal muscle tissues [8-10], together with indirectly function targeting at the somatotropic axis by altering secretions of both growth hormone and insulin-like growth factor $[11,12]$. Estrogen directly affects the structure of musculoskeletal tissues, such as muscle and ligament [13]. Restoring normal estrogen levels helps to restore cellular redox and glucose homeostasis in skeletal muscle. Estrogen improves muscle mass and muscle strength. Women suffered from accelerated muscle loss and decrease in muscle strength and function when they enter menopause since women in the menopausal period have lower circulating levels of estrogen and progesterone [14]. Muscle mass has been proved to be increased with hormone replacement therapy in postmenopausal women, regardless of exercise [15]. As an estrogen-dependent tumor, breast cancer cells will accelerate growth under the action of estrogen. Adjuvant endocrine therapy, for early breast cancer patients, consists of taking drugs with the function of antagonists of estrogen and progesterone could stop the proliferation of breast cancer cells. A breast cancer mouse model study proved that aromatase inhibitors, as a blocker of estrogen biosynthesis used as standard endocrine therapy, cause muscle weakness and bone loss [16]. Prevention of aromatase inhibitors-induced osteoclastic bone resorption with the bisphosphonate could attenuate the prevalence of bone metastases and improve muscle function in the mouse model. Nevertheless, the function of estrogen on human skeletal muscle was not clearly stratified due to the impact of many confounding differences, such as age, quality of life, dietary behavior, and intensity of exercise. Several studies researched the progesterone effects on skeletal muscle mass and its function. Progesterone receptor is found to be present in skeletal muscle cells and some functions of this steroid hormone in skeletal muscle tissue are studied [17]. Previous studies proved that treatment with progesterone has no impact on the ability of the skeletal muscle to oxidize lipids [18]. However, the relationship between progesterone and skeletal muscle mass and strength in breast cancer patients is not clearly stated.

Previous studies proved that hormones significantly influence body visceral adipose tissue distribution and visceral adipose differentiation [19]. Fat tissue is the original source of many proinflammatory cytokines in patients with more adipose tissue. Estrogen and its receptors regulate various aspects of glucose together with lipid metabolism. The decrease of estrogen is common during the menopausal period with characteristics of lipid profile variations and predominant visceral fat accumulation. The absence of estrogen has a significant impact on obesity in the menopausal period in women. Increased levels of estrogen because of excessive aromatization activity of the fat tissue and adipokines derived from fat tissue contribute to the risk to develop into breast cancer in obese women [20]. Obesity-related estrogen is a single independent prognostic factor for breast cancer patients [20]. Patients' large breast volume is proportional to the visceral adipose tissue deposits in obese premenopausal patients [21]. In postmenopausal breast cancer patients, a high visceral fat area was significantly associated with shorter distant DFS [22]. The association of adipose tissue and breast cancer is correlated to the imbalance of hormones, that is, estrogen (estrone and estradiol) [23]. In a clinical study recruiting totally 16608 women, breast cancer incidence was higher in the estrogen plus progestin group and more commonly occurred in lymph node-positive patients [24]. Aromatase mediates the crosstalk of obesity-associated inflammation and hormone alterations in patients with breast cancer [25]. The association of extended adjuvant endocrine therapy with obesity for patients with nonmetastatic breast cancer is still not clearly stated.

Thus, our study aims to investigate the association of extended adjuvant endocrine therapy with DFS, muscle mass, muscle strength, and visceral adipose tissue in patients with nonmetastatic breast cancer. Our hypothesis is that 10 years of adjuvant endocrine therapy could prolong the DFS, but decrease the muscle mass and muscle strength and increase adipose tissue at the same time.

\section{Patients and Methods}

2.1. Study Patients. 90 patients with nonmetastatic breast cancer aged between 60 and 65 years old were prospectively recruited in this study, compromising a cohort of subjects that receive at least 5 years of adjuvant endocrine therapy. Patients were recruited from July 2017 to March 2020. We selected patients with a random number table. Inclusion criteria are breast cancer patients receiving already 5 years or 10 years of adjuvant endocrine therapy, with age of 60-65 years. Patients were not subjected to radiotherapy. Exclusion criteria included patients with diabetes, metabolic syndrome, tuberculous, rheumatoid arthritis, gout, systematic inflammatory disease, cerebrovascular disease, liver or renal insufficiency, cardiac insufficiency, autoimmune disease, or other severe systemic diseases. All patients were not allowed to receive steroids prior to this study. The study was approved by the Ethics Committee of Hubei Ezhou Central Hospital. All patients signed the written informed consent.

2.2. Baseline Characteristics. We recorded patients' baseline clinical data, including age, weight, body mass index (BMI), skeletal muscle index, visceral adipose index, tumor size, lymphatic invasion, number of metastatic lymph nodes, estrogen receptor (ER) status, progesterone receptor (PR) status, human epidermal growth factor receptor 2 (Her-2) status, adjuvant endocrine therapy, previous chemotherapy, target therapy, and surgery method (Table 1).

2.3. Body Composition Analysis. We evaluated body composition including skeletal muscle mass and visceral adipose area at the third lumbar vertebrae (L3) level using abdominal CT scan using sliceOmatic software (version 5.0, Tomovision, Montreal, Canada) both at baseline and after $5 \mathrm{y}$ or $10 \mathrm{y}$ of endocrine treatment. The skeletal muscle was colored with typical Hounsfield units (HU) ranging from -29 to 150 and visceral adipose tissue was colored with $\mathrm{HU}$ ranging from -150 to -50 (Figure 1). The normalized skeletal muscle 
index and visceral adipose index were calculated by division of square of height. Skeletal muscle index = skeletal muscle mass $/$ height $^{2}\left(\mathrm{~cm}^{2} / \mathrm{m}^{2}\right)$. Visceral adipose index $=$ visceral adipose area/height ${ }^{2}\left(\mathrm{~cm}^{2} / \mathrm{m}^{2}\right)$.

2.4. Muscle Strength and Gait Speed. Muscle strength was measured using handgrip strength. For females, handgrip strength less than $20 \mathrm{~kg}$ was regarded as abnormal [26]. A common gait speed test is called the 6-meter usual walking speed test. A cut-off of $\leq 0.8 \mathrm{~m} / \mathrm{s}$ is regarded as abnormal by EWGSOP2 [26].

2.5. The Food Frequency Questionnaire (FFQ). Patients' dietary behavior was assessed using the food frequency questionnaire (FFQ). Patients were asked to do a 24-hour dietary recall. Patients' energy intake, protein, fat, and carbohydrate intake were recorded.

2.6. Statistical Methods. We did all statistical analysis using SPSS 23 (IBM, Armonk, NY). Descriptive statistical analyses of baseline characteristics were conducted for all continuous variables by mean values (standard deviation) and categorical variables by numbers (percentage). Comparisons of skeletal muscle index, visceral adipose index, muscle strength, gait speed between patients with 5 years of adjuvant endocrine therapy and 10 years of adjuvant endocrine therapy were made using Student's $t$ test with $P<0.05$ considered as statistically significant. Patients' dietary behaviors between the two groups were also compared using Student's $t$ test.

\section{Results}

3.1. Baseline Characteristics. Patients' age, body weight, and BMI did not differ between the two groups (Table 1). Patients baseline tumor size, lymphatic invasion, number of metastatic lymph nodes, estrogen receptor (ER)/progesterone receptor (PR) status, human epidermal growth factor receptor 2 (Her-2) status, endocrine therapy, and surgery are summarized in Table 1 . The median age in 5 years of endocrine therapy group was 62.5 years versus 62.8 years in the 10 years of adjuvant endocrine therapy group $(P=0.521)$. The average weight in 5 years of endocrine therapy group was $72.2 \mathrm{~kg}$ versus $72.6 \mathrm{~kg}$ in the 10 years of adjuvant endocrine therapy group $(P=0.529)$. The average BMI in 5 years of endocrine therapy group was $26.1 \mathrm{~kg} / \mathrm{m}^{2}$ versus $26.3 \mathrm{~kg} / \mathrm{m}^{2}$ in the 10 years of adjuvant endocrine therapy group $(P=0.809)$, both falling within the overweight range proposed by the World Health Organization.

\subsection{Impact of 5 Years or 10 Years of Adjuvant Endocrine} Therapy on DFS. An extended adjuvant endocrine therapy into 10 years could translate into DFS benefit. Patients with 10 years of adjuvant endocrine therapy had better DFS compared with patients with 5 years of adjuvant endocrine therapy (123.8 vs. 102.2 months, $P=0.038$ ) (Figure 2).
3.3. Impact of 5 Years or 10 Years of Adjuvant Endocrine Therapy on Body Composition. Patients' baseline skeletal muscle index or visceral fat did not have a statistical difference (Table 1). Breast cancer patients receiving 10 years of adjuvant endocrine therapy had less skeletal muscle and more visceral fat compared with patients receiving 5 years of adjuvant endocrine therapy. The skeletal muscle index in 10 years endocrine therapy group was $55.0 \pm 3.2 \mathrm{~cm}^{2} / \mathrm{m}^{2}$ versus $28.9 \pm 2.9 \mathrm{~cm}^{2} / \mathrm{m}^{2}$ in the 5 years of adjuvant endocrine therapy group $\left(28.9 \pm 2.9\right.$ vs. $\left.55.0 \pm 3.2 \mathrm{~cm}^{2} / \mathrm{m}^{2}, P=0.042\right)$ (Figure 3 ). The visceral fat in 10 years endocrine therapy group was $28.9 \pm 2.9 \mathrm{~cm}^{2} / \mathrm{m}^{2}$ versus $55.0 \pm 3.2 \mathrm{~cm}^{2} / \mathrm{m}^{2}$ in the 5 years of adjuvant endocrine therapy group $(28.9 \pm 2.9$ vs. $55.0 \pm 3.2 \mathrm{~cm}^{2} / \mathrm{m}^{2}, P=0.011$ ) (Figure 4 ).

3.4. Impact of 5 Years or 10 Years of Adjuvant Endocrine Therapy on Muscle Strength and Gait Speed. Patients' muscle strength was defined by handgrip strength. The muscle strength in the two groups did not differ, which was $27.6 \pm 2.3 \mathrm{~kg}$ in 5-year endocrine therapy group and $21.9 \pm 1.5 \mathrm{~kg}$ in the 10 -year endocrine therapy group $(P=0.329)$ (Table 2$)$. The gait speed of $6 \mathrm{~m}$ walking usual pace in two groups did not differ, which was $4.4 \pm 0.1 \mathrm{~s}$ in 5 year endocrine therapy group and $4.6 \pm 0.1 \mathrm{~s}$ in the 10 -year endocrine therapy group $(P=0.798)$ (Table 2 ).

3.5. Comparison of Patients Dietary Behaviors in 5 Years or 10 Years of Adjuvant Endocrine Therapy. Patients took a 24-hour dietary recall using the FFD (Table 3). Patients' energy intakes were $1942.1 \pm 45.5 \mathrm{kcal}$ in the 5 -year endocrine therapy group versus $1973.1 \pm 76.6 \mathrm{kcal}$ in the 10 -year endocrine therapy group $(P=0.412)$. Patients' protein intakes were $65.1 \pm 9.9 \mathrm{~g}$ in the 5-year endocrine therapy group versus $64.1 \pm 9.7 \mathrm{~g}$ in the 10-year endocrine therapy group $(P=0.221)$. Patients' fat intakes were $50.9 \pm 5.2 \mathrm{~g}$ in the 5-year endocrine therapy group versus $48.7 \pm 8.2 \mathrm{~g}$ in the 10 -year endocrine therapy group $(P=0.756)$. Patients' carbohydrate intakes were $296.7 \pm 43.1 \mathrm{~g}$ in the 5-year endocrine therapy group versus $301.1 \pm 31.8 \mathrm{~g}$ in the 10 -year endocrine therapy group $(P=0.109)$.

\section{Discussion}

Our study was the first to evaluate the impact of extended endocrine therapy on patients' DFS, body composition, muscle strength, and gait speed. We proved that patients receiving 10 years of endocrine had DFS benefit, which is in accordance with a previous study [27]. However, this 10year therapy could lead to patients' decline in muscle mass and raise in visceral adipose even though no significant difference in muscle strength and gait speed were observed. Our patients also did a FFQ, which could rule out the impact of dietary on patients' muscle and fat.

Muscle maintenance is a very important issue for cancer patients. Numerous studies have indicated that cancer patients with muscle loss had worse survival [28-32]. In patients with stages I-III of colorectal cancer, muscle wasting is an independent risk factor for all-cause mortality, regardless of other body composition parameters [28]. In a 
TABle 1: Patients baseline characteristics.

\begin{tabular}{|c|c|c|c|}
\hline & $\begin{array}{c}\text { Patients with } 5 \text { years of adjuvant endocrine } \\
\text { therapy }(n=45)\end{array}$ & $\begin{array}{c}\text { Patients with } 10 \text { years of adjuvant endocrine } \\
\text { therapy }(n=45)\end{array}$ & $\begin{array}{c}P \\
\text { value }\end{array}$ \\
\hline Age (median) & 62.5 & 62.8 & 0.521 \\
\hline Weight $(\mathrm{kg})$ & 72.2 & 72.6 & 0.529 \\
\hline BMI $\left(\mathrm{kg} / \mathrm{m}^{2}\right)$ & 26.1 & 26.3 & 0.809 \\
\hline Muscle mass index $\left(\mathrm{cm} / \mathrm{m}^{2}\right)$ & 61.5 & 64.1 & 0.217 \\
\hline Visceral adipose index $\left(\mathrm{cm} / \mathrm{m}^{2}\right)$ & 25.1 & 26.9 & 0.176 \\
\hline \multicolumn{4}{|l|}{ Tumor size } \\
\hline $\mathrm{T} 1$ & 37 & 32 & \\
\hline $\mathrm{T} 2$ & 8 & 13 & \\
\hline \multicolumn{4}{|l|}{ Lymphatic invasion } \\
\hline No & 35 & 33 & \\
\hline N1 & 10 & 12 & \\
\hline \multicolumn{4}{|l|}{ Number of metastatic lymph nodes } \\
\hline M0 & 45 & 45 & \\
\hline \multicolumn{4}{|l|}{ ER/PE status } \\
\hline $\mathrm{ER}+/ \mathrm{PR}+$ & 30 & 27 & \\
\hline $\mathrm{ER}+/ \mathrm{PR}-$ & 9 & 6 & \\
\hline $\mathrm{ER}-/ \mathrm{PR}+$ & 6 & 7 & \\
\hline $\mathrm{ER}-/ \mathrm{PR}-$ & 0 & 5 & \\
\hline \multicolumn{4}{|l|}{ Her-2 status } \\
\hline Positive & 15 & 23 & \\
\hline Negative & 30 & 22 & \\
\hline \multicolumn{4}{|l|}{ Adjuvant endocrine therapy } \\
\hline Anastrozole & 8 & 7 & \\
\hline Letrozole & 13 & 16 & \\
\hline Tamoxifen & 12 & 10 & \\
\hline Exemestane & 12 & 12 & \\
\hline \multicolumn{4}{|l|}{ Previous chemotherapy } \\
\hline Doxorubicin + cyclophosphamide & 19 & 18 & \\
\hline Docetaxel + cyclophosphamide & 19 & 22 & \\
\hline Docetaxel + carboplatin & 7 & 5 & \\
\hline \multicolumn{4}{|l|}{ Target therapy (transtuzumab) } \\
\hline Yes & 15 & 21 & \\
\hline No & 30 & 24 & \\
\hline \multicolumn{4}{|l|}{ Surgery } \\
\hline Modified radical mastectomy & 11 & 13 & \\
\hline Breast conservation & 34 & 32 & \\
\hline
\end{tabular}

BMI: body mass index; ER: estrogen receptor; PR: progesterone receptor; Her-2: human epidermal growth factor receptor 2.

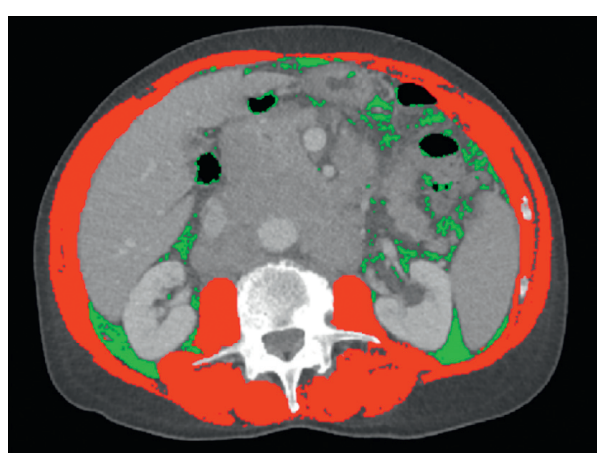

(a)

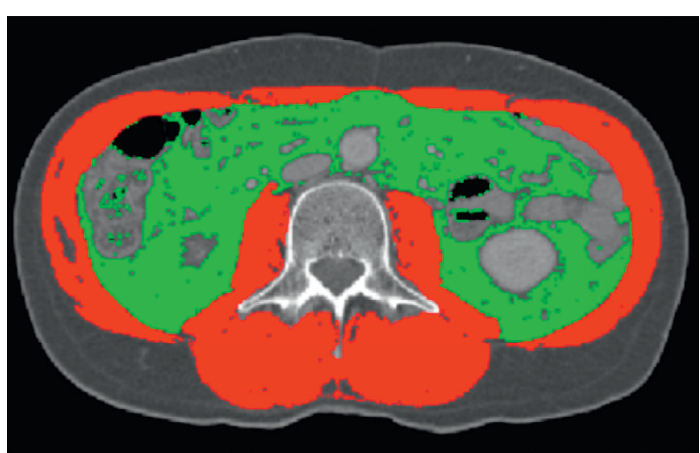

(b)

Figure 1: Example of body composition analysis using SliceOmatic software. The red zone represents muscle mass and green zone represents visceral adipose tissue. (a) Patients with low fat. (b) Patients with high fat. 


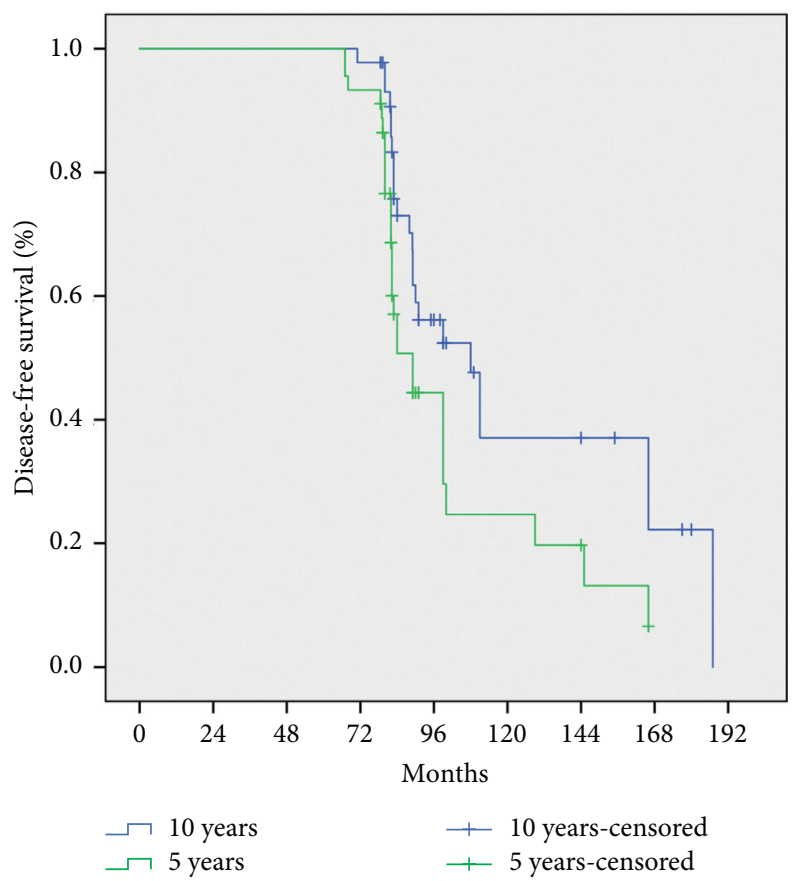

FiguRe 2: Kaplan-Meier curve of breast cancer patients receiving 5 years or 10 years of adjuvant endocrine therapy. Patients receiving 10 years of adjuvant endocrine therapy had better DFS compared to patients receiving 5 years of adjuvant endocrine therapy (123.8 vs. 102.2 months, $P=0.038$ ).

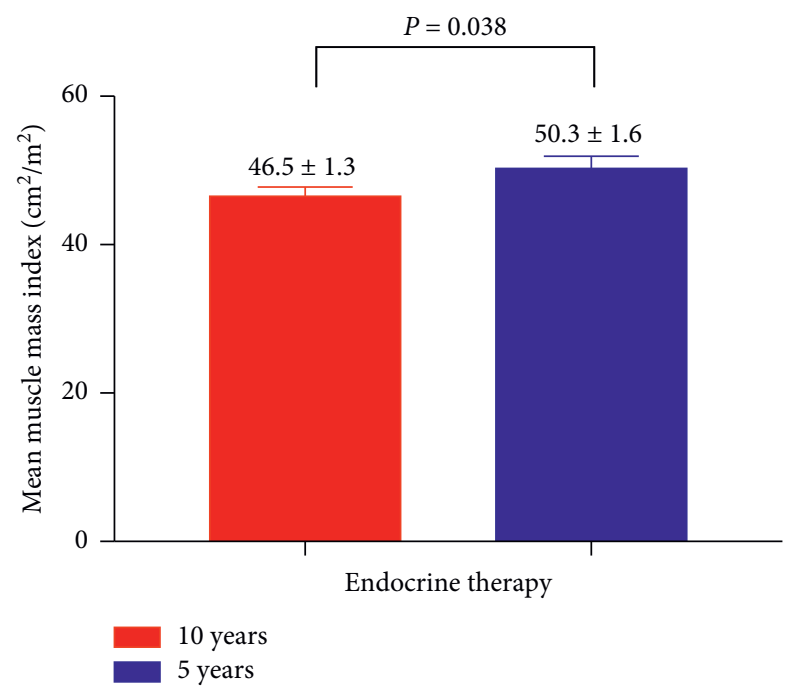

Figure 3: Comparison of muscle mass index of breast cancer patients receiving 5 years or 10 years of adjuvant endocrine therapy. Breast cancer patients receiving 5 years of adjuvant endocrine therapy had higher muscle index compared with breast cancer patients receiving 10 years of adjuvant endocrine therapy $\left(50.3 \pm 1.6\right.$ vs. $\left.46.5 \pm 1.3 \mathrm{~cm}^{2} / \mathrm{m}^{2}, P=0.042\right)$.

retrospective study of 2042 patients undergoing radical prostatectomy of prostate cancer, low muscle mass was approved to be associated with increased risks of recurrence and mortality, no matter the BMI [30]. A retrospective study of 231 metastatic gastric cancer patients proved that skeletal muscle loss during chemotherapy was associated with shorter overall survival (OS) [31]. Over one-third of newly diagnosed patients with nonmetastatic breast cancer patients were found to have low muscle radiodensity [32]. In an observational study of 3241 patients diagnosed with nonmetastatic breast cancer, low muscle radiodensity and high total adipose tissue were statistically significantly related to overall mortality [32].

Adipose tissue contributes to cancer development and is negatively associated with prognosis in several cancer types [33-37]. There was an association between obesity and breast cancer risk in postmenopausal ER/PR-positive patients in many clinical studies [35-37]. Obesity was 


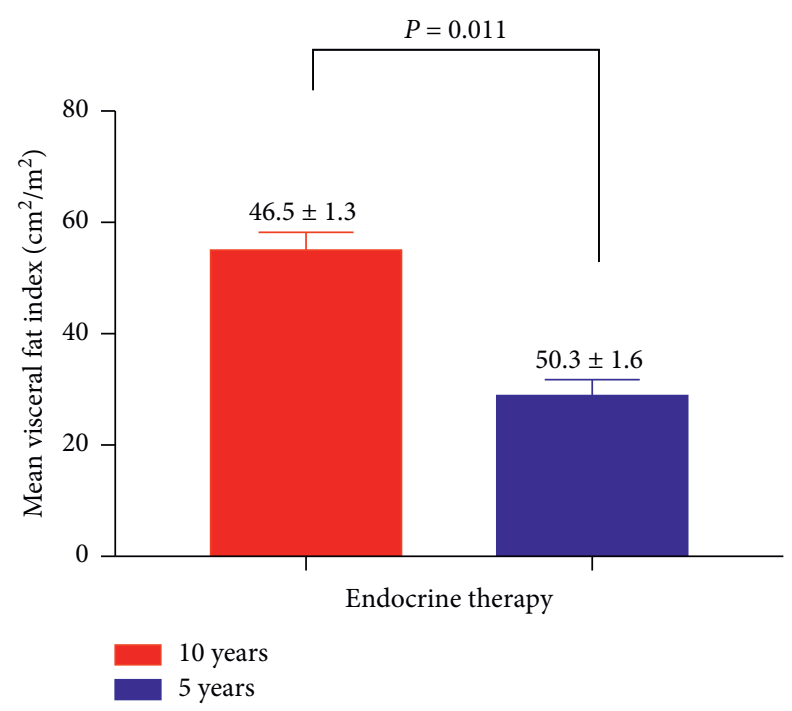

Figure 4: Comparison of visceral fat of breast cancer patients receiving 5 years or 10 years of adjuvant endocrine therapy. Breast cancer patients receiving 5 years of adjuvant endocrine therapy had less visceral fat compared with breast cancer patients receiving 10 years of adjuvant endocrine therapy $\left(28.9 \pm 2.9\right.$ vs. $\left.55.0 \pm 3.2 \mathrm{~cm}^{2} / \mathrm{m}^{2}, P=0.011\right)$.

TABLE 2: Patients muscle strength and gait speed in the two groups.

\begin{tabular}{|c|c|c|c|}
\hline Mean \pm standard deviation & $\begin{array}{c}\text { Patients with } 5 \text { years of adjuvant endocrine } \\
\text { therapy }(n=45)\end{array}$ & $\begin{array}{c}\text { Patients with } 10 \text { years of adjuvant endocrine } \\
\text { therapy }(n=45)\end{array}$ & $\begin{array}{c}P \\
\text { value }\end{array}$ \\
\hline Muscle strength $(\mathrm{kg})$ & $27.6 \pm 2.3$ & $21.9 \pm 1.5$ & 0.329 \\
\hline $\begin{array}{l}\text { Gait speed of } 6 \mathrm{~m} \text { walking usual } \\
\text { pace }(\mathrm{s})\end{array}$ & $4.4 \pm 0.1$ & $4.6 \pm 0.1$ & 0.798 \\
\hline
\end{tabular}

TABle 3: Patients dietary behaviors in two groups.

\begin{tabular}{lccc}
\hline & $\begin{array}{c}\text { Patients with 5 years of adjuvant endocrine therapy } \\
(n=45)\end{array}$ & $\begin{array}{c}\text { Patients with 10 years of adjuvant endocrine therapy } \\
(n=45)\end{array}$ & $\begin{array}{c}P \text { value } \\
\text { Energy (kcal) }\end{array}$ \\
Protein (g) & $1942.1 \pm 45.5$ & $673.1 \pm 76.6$ & 0.412 \\
Fat (g) & $65.1 \pm 9.9$ & $48.7 \pm 9.7$ & 0.221 \\
Carbohydrate(g) & $50.9 \pm 5.2$ & $301.1 \pm 31.8$ & 0.756 \\
\hline
\end{tabular}

associated with shorter DFS and increased mortality rate in both premenopausal and postmenopausal breast cancer patients. Women who had a BMI higher than $40 \mathrm{~kg} / \mathrm{m}^{2}$ had a 2 -fold higher risk of mortality rate in comparison with breast cancer patients with normal BMI (RR, 2.12) [38]. Other studies proved that higher adipose tissue was associated with shorter distant DFS and OS, larger tumors, positive lymph node status, and triple-negative tumor subtype [39, 40].

Estrogen and progesterone are the two primary female sex hormones. They are produced mainly in ovaries and produced or converted into forms of estrogen in fat tissue. Estrogen balance is essential for achieving and maintaining muscle and fat loss. Estrogen significantly facilitates adipose tissue depots and its function [41]. Estrogen protects against accumulated visceral adipose through its function to suppress appetite and raise energy expenditure. A clinical study of 294 patients with invasive breast cancer proved that invasive breast cancer mainly occurs adjacent to breast adipose tissue, irrespective of the tumor volume and patients' ER/PR status [42]. A previous trial ATLAS proved that patients treated with tamoxifen for ten years have reduced risk of breast cancer recurrence and mortality compared to those treated with tamoxifen for only 5 years [43]. However, more side effects occurred, such as loss of bone mineral density [44]. There is no published article regarding the impact of extended adjuvant endocrine therapy on body composition, muscle strength, and gait speed. Our study was the first to prove that 10 years of adjuvant could decrease patients' muscle mass index and increase their adipose tissue even though they had almost the same daily energy intakes compared with patients with 5 years of adjuvant therapy.

Our study also has limitations. Our sample size is not large, which means the conclusions still need to be validated in a larger-scale clinical trial. Our study is an observational cohort prospective study; thus, we could not rule out confounding factors that could impact muscle or fat, such as 
exercise. However, we tried our best to do a FFQ, which rule out the impact of dietary on muscle and fat. Patients' baseline characteristics including age, sex, and BMI did not reach significant difference. However, patients with the same BMI may have different adipose tissue compartments and muscle mass. Our study was the first to look further into the impact of endocrine therapy on body composition in breast cancer patients.

\section{Conclusion}

In conclusion, breast cancer patients with 10 years of adjuvant endocrine therapy had better DFS, but with more muscle loss and adipose tissue deposits compared with patients receiving 5 years of adjuvant endocrine therapy.

\section{Data Availability}

The datasets analyzed during the present study are available from the corresponding author on reasonable request.

\section{Ethical Approval}

The studies involving human subjects were reviewed and approved by the Medical Ethics Review Committee of Hubei Ezhou Central Hospital.

\section{Consent}

All patients who participated in our observational cohort study provided written informed consent before entering the study.

\section{Conflicts of Interest}

The authors declared that there are no conflicts of interest regarding this article.

\section{Authors' Contributions}

Zhaoshi Yu designed the study; Ruyi Hu collected the data and performed the analysis; Ruyi Hu and Xinran Cheng drafted the manuscript. All the other authors read and agreed on the final manuscript. Ruyi Hu and Xinran Cheng contributed equally.

\section{Acknowledgments}

The study was supported by Hubei Ezhou Central Hospital.

\section{References}

[1] C. Ou, H. Li, J. H. Liu et al., "Meta-analysis of transforming growth factor $\beta$ receptor I 6A/9A gene polymorphism and breast cancer risk: the picture remains murky," Biomarkers :Biochemical Indicators of Exposure, Response, and Susceptibility to Chemicals, vol. 20, no. 6-7, pp. 487-494, 2015.

[2] P. Ssentongo, J. A. Lewcun, X. Candela et al., "Regional, racial, gender, and tumor biology disparities in breast cancer survival rates in Africa: a systematic review and meta-analysis," PLoS One, vol. 14, no. 11, Article ID e0225039, 2019.
[3] A. R. Ferreira, A. Palha, L. Correia et al., "Treatment adoption and relative effectiveness of aromatase inhibitors compared to tamoxifen in early breast cancer: a multi-institutional observational study," The Breast, vol. 37, pp. 107-113, 2018.

[4] "Tamoxifen for early breast cancer: an overview of the randomised trials. Early breast cancer trialists' collaborative group," Lancet, vol. 351, no. 9114, pp. 1451-1467, 1998.

[5] "Effects of chemotherapy and hormonal therapy for early breast cancer on recurrence and 15-year survival: an overview of the randomised trials," Lancet, vol. 365, no. 9472, pp. 1687-1717, 2005.

[6] F. Petrelli, A. Coinu, M. Cabiddu, M. Ghilardi, V. Lonati, and S. Barni, "Five or more years of adjuvant endocrine therapy in breast cancer: a meta-analysis of published randomised trials," Breast Cancer Research and Treatment, vol. 140, no. 2, pp. 233-240, 2013.

[7] L. B. Tankó, L. Movsesyan, O. L. Svendsen, and C. Christiansen, "The effect of hormone replacement therapy on appendicular lean tissue mass in early postmenopausal women," Menopause, vol. 9, no. 2, pp. 117-121, 2002.

[8] M. Brown, S. J. Birge, and W. M. Kohrt, "Hormone replacement therapy does not augment gains in muscle strength or fat-free mass in response to weight-bearing exercise," The Journals of Gerontology Series A: Biological Sciences and Medical Sciences, vol. 52A, no. 3, pp. B166-B170, 1997.

[9] D. R. Taaffe, A. B. Newman, C. L. Haggerty et al., "Estrogen replacement, muscle composition, and physical function: the health ABC study," Medicine \& Science in Sports \& Exercise, vol. 37, no. 10, pp. 1741-1747, 2005.

[10] S. Lemoine, P. Granier, C. Tiffoche, F. O. Rannou-Bekono, M.-L. Thieulant, and P. Delamarche, "Estrogen receptor alpha mRNA in human skeletal muscles," Medicine \& Science in Sports \& Exercise, vol. 35, no. 3, pp. 439-443, 2003.

[11] D. A. Bemben and D. B. Langdon, "Relationship between estrogen use and musculoskeletal function in postmenopausal women," Maturitas, vol. 42, no. 2, pp. 119-127, 2002.

[12] K. E. Friend, M. L. Hartman, S. S. Pezzoli, J. L. Clasey, and M. O. Thorner, "Both oral and transdermal estrogen increase growth hormone release in postmenopausal women-a clinical research center study," The Journal of Clinical Endocrinology \& Metabolism, vol. 81, no. 6, pp. 2250-2256, 1996.

[13] N. Chidi-Ogbolu and K. Baar, "Effect of estrogen on musculoskeletal performance and injury risk," Frontiers in Physiology, vol. 9, p. 1834, 2018.

[14] L. M. Kurina, M. Gulati, S. A. Everson-Rose et al., "The effect of menopause on grip and pinch strength: results from the Chicago, Illinois, site of the study of women's health across the nation," American Journal of Epidemiology, vol. 160, no. 5, pp. 484-491, 2004.

[15] S. Sipilä, D. R. Taaffe, S. Cheng, J. Puolakka, J. Toivanen, and H. Suominen, "Effects of hormone replacement therapy and high-impact physical exercise on skeletal muscle in postmenopausal women: a randomized placebo-controlled study," Clinical Science, vol. 101, no. 2, pp. 147-157, 2001.

[16] L. E. Wright, A. A. Harhash, W. M. Kozlow et al., "Aromatase inhibitor-induced bone loss increases the progression of estrogen receptor-negative breast cancer in bone and exacerbates muscle weakness in vivo," Oncotarget, vol. 8, no. 5, pp. 8406-8419, 2017.

[17] N. Uotinen, R. Puustinen, S. Pasanen et al., "Distribution of progesterone receptor in female mouse tissues," General and Comparative Endocrinology, vol. 115, no. 3, pp. 429-441, 1999.

[18] Y. J. Kim, A. Tamadon, H. T. Park, H. Kim, and S.-Y. Ku, "The role of sex steroid hormones in the pathophysiology and 
treatment of sarcopenia," Osteoporosis and Sarcopenia, vol. 2, no. 3, pp. 140-155, 2016.

[19] F. Lizcano and G. Guzmán, "Estrogen deficiency and the origin of obesity during menopause," BioMed Research International, vol. 2014, Article ID 757461, 11 pages, 2014.

[20] A. Engin, "Obesity-associated breast cancer: analysis of risk factors," Obesity and Lipotoxicity, vol. 960, pp. 571-606, 2017.

[21] P. M. Janiszewski, T. J. Saunders, and R. Ross, "Breast volume is an independent predictor of visceral and ectopic fat in premenopausal women," Obesity, vol. 18, no. 6, pp. 1183-1187, 2010.

[22] T. Iwase, T. Sangai, T. Nagashima et al., "Impact of body fat distribution on neoadjuvant chemotherapy outcomes in advanced breast cancer patients," Cancer Medicine, vol. 5, no. 1, pp. 41-48, 2016.

[23] A. Lukanova, E. Lundin, A. Zeleniuch-Jacquotte et al., "Body mass index, circulating levels of sex-steroid hormones, IGF-I and IGF-binding protein-3: a cross-sectional study in healthy women," European Journal of Endocrinology, vol. 150, no. 2, pp. 161-171, 2004.

[24] R. T. Chlebowski, G. L. Anderson, M. Gass et al., "Estrogen plus progestin and breast cancer incidence and mortality in postmenopausal women," JAMA, vol. 304, no. 15, pp. 1684-1692, 2010.

[25] K. Subbaramaiah, P. G. Morris, X. K. Zhou et al., "Increased levels of COX-2 and prostaglandin E2 contribute to elevated aromatase expression in inflamed breast tissue of obese women," Cancer Discovery, vol. 2, no. 4, pp. 356-365, 2012.

[26] A. J. Cruz-Jentoft, G. Bahat, J. Bauer et al., "Sarcopenia: revised European consensus on definition and diagnosis," Age and Ageing, vol. 48, no. 1, pp. 16-31, 2019.

[27] L. Li, B. Chang, X. Jiang et al., "Clinical outcomes comparison of 10 years versus 5 years of adjuvant endocrine therapy in patients with early breast cancer," BMC Cancer, vol. 18, no. 1, p. 977, 2018.

[28] J. C. Brown, B. J. Caan, J. A. Meyerhardt et al., "The deterioration of muscle mass and radiodensity is prognostic of poor survival in stage I-III colorectal cancer: a populationbased cohort study (C-SCANS)," Journal of Cachexia, Sarcopenia and Muscle, vol. 9, no. 4, pp. 664-672, 2018.

[29] V. E. Baracos and L. Arribas, "Sarcopenic obesity: hidden muscle wasting and its impact for survival and complications of cancer therapy," Annals of Oncology: Official Journal of the European Society for Medical Oncology, vol. 29, 2018.

[30] S. Pak, S. Y. Park, T. J. Shin et al., "Association of muscle mass with survival after radical prostatectomy in patients with prostate cancer," Journal of Urology, vol. 202, no. 3, pp. 525-532, 2019.

[31] K. Sugiyama, Y. Narita, S. Mitani et al., "Baseline sarcopenia and skeletal muscle loss during chemotherapy affect survival outcomes in metastatic gastric cancer," Anticancer Research, vol. 38, no. 10, pp. 5859-5866, 2018.

[32] B. J. Caan, E. M. Cespedes Feliciano, C. M. Prado et al., "Association of muscle and adiposity measured by computed tomography with survival in patients with nonmetastatic breast cancer," JAMA Oncology, vol. 4, no. 6, pp. 798-804, 2018.

[33] A. J. Cozzo, A. M. Fuller, and L. Makowski, "Contribution of adipose tissue to development of cancer," Comprehensive Physiology, vol. 8, no. 1, pp. 237-282, 2017.

[34] F. Bertolini, "Adipose tissue and breast cancer progression: a link between metabolism and cancer," The Breast, vol. 22, no. 2, pp. S48-S49, 2013.
[35] A. J. White, H. B. Nichols, P. T. Bradshaw, and D. P. Sandler, "Overall and central adiposity and breast cancer risk in the sister study," Cancer, vol. 121, no. 20, pp. 3700-3708, 2015.

[36] M. Cotterchio, N. Kreiger, B. Theis, M. Sloan, and S. Bahl, "Hormonal factors and the risk of breast cancer according to estrogen- and progesterone-receptor subgroup," Cancer Epidemiology, Biomarkers \& Prevention, vol. 12, no. 10, pp. 1053-1060, 2003.

[37] S. M. Enger, R. K. Ross, A. Paganini-Hill, C. L. Carpenter, and L. Bernstein, "Body size, physical activity, and breast cancer hormone receptor status: results from two case-control studies," Cancer Epidemiology, Biomarkers \& Prevention: A Publication of the American Association for Cancer Research, vol. 9, no. 7, pp. 681-687, 2000.

[38] E. E. Calle, C. Rodriguez, K. Walker-Thurmond, and M. J. Thun, "Overweight, obesity, and mortality from cancer in a prospectively studied cohort of U.S. adults," New England Journal of Medicine, vol. 348, no. 17, pp. 1625-1638, 2003.

[39] E. R. Copson, R. I. Cutress, T. Maishman et al., "Obesity and the outcome of young breast cancer patients in the UK: the POSH study," Annals of Oncology, vol. 26, no. 1, pp. 101-112, 2015.

[40] L. Rosenberg, K. Czene, and P. Hall, “Obesity and poor breast cancer prognosis: an illusion because of hormone replacement therapy?" British Journal of Cancer, vol. 100, no. 9, pp. 1486-1491, 2009.

[41] B. F. Palmer and D. J. Clegg, "The sexual dimorphism of obesity," Molecular and Cellular Endocrinology, vol. 402, pp. 113-119, 2015.

[42] W. Zhu, S. Harvey, K. J. Macura, D. M. Euhus, and D. Artemov, "Invasive breast cancer preferably and predominantly occurs at the interface between fibroglandular and adipose tissue," Clinical Breast Cancer, vol. 17, no. 1, pp. e11-e18, 2017.

[43] C. Davies, H. Pan, J. Godwin et al., "Long-term effects of continuing adjuvant tamoxifen to 10 years versus stopping at 5 years after diagnosis of oestrogen receptor-positive breast cancer: ATLAS, a randomised trial," The Lancet, vol. 381, no. 9869, pp. 805-816, 2013.

[44] T. J. Powles, T. Hickish, J. A. Kanis, A. Tidy, and S. Ashley, "Effect of tamoxifen on bone mineral density measured by dual-energy X-ray absorptiometry in healthy premenopausal and postmenopausal women," Journal of Clinical Oncology, vol. 14, no. 1, pp. 78-84, 1996. 